\title{
Sécurité des patients: où en sommes-nous aujourd'hui?
}

\author{
Margrit Leuthold ${ }^{a}$, Dieter Conen ${ }^{b}$, Kathrin Hirter ${ }^{c}$, Enea Martinellid \\ ${ }^{a}$ Dr, directrice Fondation pour la Sécurité des patients Suisse; ${ }^{b}$ président Fondation pour la Sécurité des patients Suisse; ${ }^{c}$ vice-présidente Fondation pour la \\ Sécurité des patients Suisse; ${ }^{d}$ Dr, vice-président Fondation pour la Sécurité des patients Suisse
}

En Suisse aussi, un long chemin a été parcouru au cours de ces dernières années: parler des erreurs commises est possible, la majorité des hôpitaux disposant d'un système de déclaration d'erreurs. Cependant, il reste encore du travail: avec la croissance exponentielle des connaissances, la complexité des technologies et les mutations socio-culturelles, les améliorations obtenues ont toujours un temps de retard face aux défis sans cesse renouvelés. La Fondation pour la Sécurité des patients Suisse s'engage afin de soutenir et renforcer les efforts des entreprises et des professionnels.

Avec la publication To err is human de l'American Institute of Medicine en 2000, le monde entier a pris conscience qu'en médecine aussi, des erreurs surviennent fréquemment et que les patients peuvent être victimes de dommages dus aux soins de santé. Les études internationales montrent que près de $10 \%$ des patients hospitalisés présentent un événement indésirable $[1,2]$, dont la moitié environ est considérée

\section{Une approche prospective est nécessaire pour pouvoir éviter efficacement les effets indésirables.}

comme évitable. Environ 0,1\% des patients hospitalisés décèdent d'un événement indésirable potentiellement évitable [2]. Depuis lors, on assiste à un changement culturel lent mais continu. La «faillabilité systémique des soins de santé» est aujourd'hui souvent abordée non seulement par les professionnels de la santé, mais aussi par les patients et le public. Le regard se déplace de plus en plus de l'individu vers l'organisation, avec pour corollaire un abandon de la notion de culpabilité. La question est moins de savoir "qui» est responsable, mais plutôt "pourquoi cela a-t-il pu se produire?» ou "comment en est-on arrivé là?». De nombreux hôpitaux ont mis en place le système de déclaration d'erreur CIRS (Critical Incidence Reporting System); ce dernier permet de déclarer anonymement des erreurs ou des dommages évités de peu dans le but d'analyser systématiquement les déclarations, de les refléter dans le système et ainsi de tirer les enseignements de ces erreurs. Si l'établissement du CIRS consti- tue une base importante, son succès, autrement dit son utilité pratique, dépend toutefois essentiellement de la façon dont ce système est employé. A cet égard, ce sont surtout des paramètres comme la qualité et la fréquence de la déclaration qui s'avèrent déterminants. Qui plus est, presque tous les hôpitaux possèdent également de nos jours une équipe de gestion du risque clinique dont la mission explicite est d'accroître la sécurité des patients. Tous ces efforts vont dans la bonne direction et représentent des jalons importants vers une meilleure culture de la sécurité.

La possibilité, au sein même d'une organisation, d'analyser les erreurs ou les quasi-erreurs dépend étroitement de la culture dominante, ce qui signifie que les cadres jouent ici un rôle décisif. Si le climat de l'entreprise permet que les erreurs puissent être thématisées, que le speak-up soit encouragé (voir à ce sujet l'article de D. Schwappach et coll. dans ce numéro), que des structures comme des conférences de mortalité et de morbidité soient établies et utilisées correctement, et que l'on tienne compte non seulement des soucis et de

\section{La fondation est le centre de compétence} national pour tous les intérêts ayant trait à la sécurité des patients.

la détresse des patients concernés par un incident, mais aussi des collaborateurs comme "second victims», alors on peut dire qu'un pas décisif vers une culture de la sécurité a été franchi. Comme nous le savons cependant, ceci est encore loin d'être le cas dans tous les établissements. Les facteurs pouvant entraver 


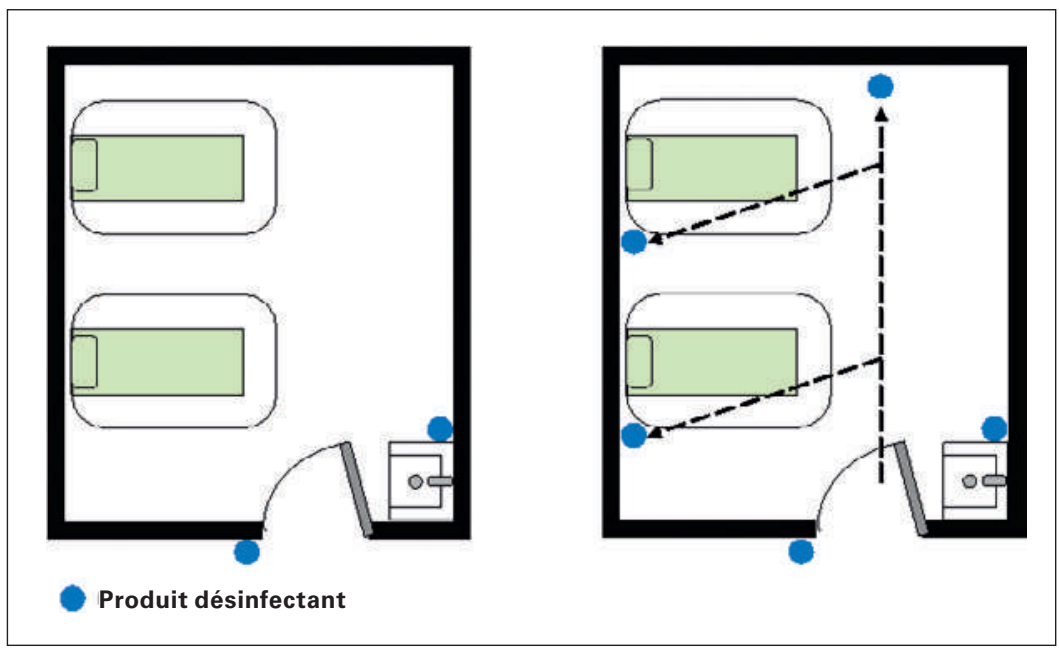

Si le distributeur se situe dans le champ de vision aux moments critiques, I'observance en matière d'hygiène des mains peut passer de 12 à $56 \%$.

cette démarche sont notamment un manque de conscience du problème chez les cadres ou une hiérarchie rigide, mais aussi une pression économique importante qui ne laisse ni le temps ni l'espace de développer une culture durable de la sécurité.

Les analyses des erreurs ou des quasi-erreurs sont le plus souvent rétrospectives. Une approche prospective est nécessaire pour pouvoir éviter efficacement les effets indésirables, c'est-à-dire pour repérer et éliminer dans le système les éventuels maillons faibles qui favorisent le risque d'erreurs.

Des interventions - qu'elles soient isolées ou qu'il s'agisse d'un ensemble d'interventions - développées et mises en œuvre pour des problématiques spécifiques constituent une approche répandue pour améliorer la sécurité des patients. Des études internationales montrent que de telles mesures permettent d'obtenir des résultats convaincants, du moins à court terme. L'approche interdisciplinaire ainsi que des trainings et des formations spécifiques permettent égale-

\section{Treize pour cent des Suisses indiquent ainsi avoir récemment été victime d'une erreur thérapeutique.}

ment d'améliorer la collaboration et la communication au sein de l'équipe, deux composantes cruciales pour la réduction des événements indésirables évitables. Dans la mesure où ce type de programmes d'amélioration repose aussi essentiellement sur les modifications comportementales des collaborateurs, leur succès durable est cependant encore incertain. La psychologie nous enseigne et notre propre expérience quotidienne nous montre qu'il est extrêmement difficile de modifier des comportements. Un monitorage à l'issue du programme semble indispensable pour pouvoir obser- ver si les changements et les améliorations obtenus ont pu effectivement perdurer.

Une autre approche prometteuse vise à organiser l'environnement professionnel de telle sorte qu'il soit plus facile d'avoir un comportement «adéquat». Une réglementation claire des procédures et processus internes à l'établissement, la disposition et l'accessibilité du matériel, un agencement de l'environnement avec l'optimisation de facteurs comme la lumière, le bruit, etc. peuvent également contribuer de façon déterminante à réduire le nombre d'erreurs. On a ainsi pu montrer par exemple que l'observance en matière d'hygiène des mains peut passer de 12 à $56 \%$ lorsque le distributeur contenant le produit désinfectant est placé de telle façon qu'il saute immédiatement aux yeux du personnel soignant [1] (voir figure). Si le distributeur se situe dans le champ de vision aux moments critiques, il est automatiquement utilisé plus souvent par le personnel sans qu'un effort cognitif conscient soit pour autant nécessaire. Le potentiel d'une telle "prévention contextuelle» doit être à l'avenir davantage mis à profit pour améliorer la sécurité des patients.

\section{La contribution de Sécurité des patients Suisse}

Créée en 2003, la fondation est le centre de compétence national pour tous les intérêts ayant trait à la sécurité des patients. Elle cherche en premier lieu à soutenir et renforcer les prestataires de soins dans leur engagement en faveur d'une meilleure culture de la sécurité. En se fondant sur des études, elle génère un savoir et en tire de nouvelles connaissances et des recommandations d'action pratiques qui sont diffusées à travers des cours, des congrès et des formations. De plus, dans le cadre de la stratégie fédérale en matière de qualité dans le système de santé suisse, elle conduit depuis 2012 dans divers «hot spots» de la sécurité des patients des programmes pilotes «progress!» avec des établissements sélectionnés. Le premier programme «progress! La sécurité en chirurgie» s'achèvera cet été, le second programme «La sécurité de la médication aux interfaces» a débuté en 2014. Par ailleurs, deux nouveaux programmes concernant les thèmes suivants sont en cours d'élaboration: la sécurité dans le sondage vésical (voir à ce sujet l'article à la page 1358 dans ce numéro) et la sécurité de la médication dans les établissements médico-sociaux.

Dans le passé, les activités clés de la fondation concernaient avant tout les problèmes rencontrés dans les soins aigus en milieu hospitalier. Publiée en 2011 et largement financée par la FMH, l'étude de Gehring et Schwappach s'est intéressée pour la première fois aux 
événements indésirables (dits «hot spots») et à la sécurité dans les soins de base [2]. Le rôle du pharmacien d'officine dans la sécurité du traitement médicamenteux, notamment à travers le conseil à la clientèle ayant un vécu migratoire, a également été étudié ces dernières années, et d'importants enseignements concernant des risques particuliers ont pu en être tirés [3]. Avec le transfert d'interventions invasives même importantes et d'autres traitements jadis traditionnellement réalisés en milieu hospitalier, le domaine ambulatoire acquiert une importance croissante et de nouvelles questions de sécurité ne manquent pas de surgir. Là encore, la fondation entend bien jouer un rôle actif à l'avenir.

Qui plus est, nous effectuons pour la première fois cette année un état des lieux concernant les aspects liés à la sécurité des patients en psychiatrie et dans les soins de longue durée. Conjointement avec des experts des disciplines les plus diverses, nous discutons des particularités dans chaque domaine et en tirerons des priorités d'action et des projets correspondants.

Les cours proposés par Sécurité des patients Suisse "A la fois auteur et victime», "Error and risk analysis» ainsi que "La communication avec les patients et leurs proches à la suite d'un incident» continuent à bénéficier d'une demande soutenue. De plus, un nouveau module destiné aux instances dirigeantes des hôpitaux est actuellement en cours d'élaboration.

Initiée et réalisée pour la première fois par Sécurité des patients Suisse, la semaine d'action 2015 offre aux prestataires de soins une plate-forme pour présenter le travail déjà accompli.

Elle vise, en coopération avec de nombreux acteurs, à attirer l'attention sur l'amélioration de la sécurité des patients et à informer le public. Diverses manifestations présenteront les thématiques centrales pour la sécurité des patients, ce que les prestataires de soins font pour favoriser la sécurité des patients, ce qu'il est urgent d'entreprendre ainsi que les efforts supplémentaires à fournir.

L'objectif de cette semaine d'action est de faire connaître encore plus largement le thème de la sécurité des patients autant dans les établissements de santé qu'auprès de la population, et de souligner son importance pour la santé publique.

La semaine d'action est une occasion unique d'informer les patients et leurs proches sur les aspects impor- tants pour la sécurité. Il s'agit là à n'en pas douter d'une problématique centrale pour tous les individus: selon une étude récente, treize pour cent des Suisses indiquent ainsi avoir récemment été victime d'une erreur thérapeutique; c'est en Angleterre que ce chiffre est le plus bas (7,8\%), et en Norvège qu'il est le plus élevé $(21,1 \%)[4]$.

\section{Perspectives}

La sécurité des patients est et demeure un thème essentiel dans la médecine moderne.

Face à la croissance exponentielle des connaissances, aux technologies et aux procédures de plus en plus complexes ainsi qu'aux évolutions socio-culturelles en médecine, les améliorations obtenues sont cependant toujours en retard par rapport aux défis toujours nouveaux qui surgissent.

La Fondation pour la Sécurité des patients Suisse veillera également à l'avenir à relever les défis actuels et futurs dans l'ensemble de la chaîne de traitement médical et à contribuer, dans le cadre de ses possibilités, à ce que la médecine ne soit pas seulement meilleure (et plus chère), mais aussi plus sûre.

Avec le projet de loi devant servir de base à un «Centre national pour la qualité des soins et la sécurité des patients» lancé l'année dernière, cette thématique est désormais à l'ordre du jour au niveau politique. Afin de pouvoir continuer à apporter sa contribution avec encore plus d'efficacité, nous espérons que la fondation bénéficiera à l'avenir d'un financement approprié à l'importance de sa mission.

Nous remercions la FMH d'avoir, dans le cadre de notre première semaine d'action, dédié l'intégralité de ce numéro à la sécurité des patients.

\section{Références}

1 Birnbach DJ, Nevo I, Scheinman SR, Fitzpatrick M, Shekhter I, Lombard JL. Patient safety begins with proper planning: a quantitative method to improve hospital design. Qual Saf Health Care. 2010;19(5):462-5.

2 Gehring K, Schwappach D, Battaglia M, Buff R, Huber F, Sauter P, et al. Frequency of and Harm Associated With Primary Care Safety Incidents. Am J Manag Care. 2012;18(9):e323-e337.

3 Schwappach D, Meyer Massetti C, Gehring K. Communication barriers in counselling foreign-language patients in public pharmacies: threats to patient safety? International Journal of Clinical Pharmacy. 2012;34(5):765-72.

4 Schwappach DLB. Risk factors for patient-reported medical errors in eleven countries. Health Expect. 2014;17(3):321-31. 